\title{
An Automated Parking Guidance System for Megacities
}

\author{
Monika Mangla \\ Research Scholar, Thapar University, Patiala, India \\ Faculty, Lokmanya Tilak College of Engineering, Navi Mumbai, India \\ E-mail: manglamona@gmail.com \\ Deepak Garg \\ Professor and Head, Bennett University, Greater Noida, India \\ E-mail: deepakgarg108@gmail.com
}

Received: 16 October 2017; Accepted: 03 December 2017; Published: 08 January 2018

\begin{abstract}
Enormous increase for vehicles in the megacities, with limited parking creates a serious issue. In order to handle the issue, many cities have adopted the guided parking as a part of Intelligent Transportation System (ITS). The current ITS is continuously evolving to incorporate the required issues. ITS communicates among vehicles and parking facilities and shares the information of interest. Thereafter ITS employs dynamic information obtained from vehicles for guiding the parking. In the current work, authors have suggested two functions for parking guidance in this study. Using these functions, central server uses this dynamic information obtained from sensory networks and uses the same to suggest parking to the driver. The driver, upon receiving the suggestion, in turn may reserve the suggested parking or may choose to decline the suggestion based on his personal experience. The proposed approach considers various parameters to evaluate effectiveness of the guided parking. During simulation, these parameters have been demonstrated and it is observed that the proposed system outperforms the existing system in literature.
\end{abstract}

Index Terms - Intelligent Transportation System, Guided parking, parking reservation system, cost function, ICT.

\section{INTRODUCTION}

The number of cars has been continuously increasing which has become a disaster for megacities. This continuous increase in the number of vehicles poses many issues like increase in the road accidents, level of pollution and road congestions etc. All these issues are related to health of humans and environment and thus needs to be handled with utmost care and importance. Many researchers have worked towards these issues using various technologies. While surveying the literature, it is observed that sufficient work is available on route planning, location modeling, and delivery planning which all are indirectly handling the issues raised by continuous growth in the number of vehicles.

Another issue which results by continuous growth in the number of vehicles is parking management. This issue of parking management further intricate with limited parking and therefore necessitates an intelligent parking solution. With limited parking, many cars can be seen circling in order to find a parking facility, which increases the traffic and also causes energy loss, environmental pollution etc. Thus over the past few decades, many researchers have intensively worked to handle parking management in an efficient manner by providing a guided parking solution.

Many cities across the globe are already using the Intelligent Transportation System, although the ITS is still in evolution phase. The employment of latest advancements in Information and Communication Technology (ICT) further escalates the guiding parking system by providing dynamic information to the central server. Central server uses this dynamic information to suggest a parking to the requesting driver by sending a parking suggestion message. This parking suggestion message (PSM) consists of suggested parking along with its tariff, distance and direction from current location. Now the driver may choose among the parking suggestions based on his experience and knowledge. If the driver chooses amongst the suggested parking options, he may go ahead and reserve one. After a parking has been reserved by driver, it will not be allocated to anyone else until the driver approaches the parking or cancels the reservation. Although this reservation of parking calls for additional cost.

Some of the parking management systems require sharing of parking also where a particular parking may be shared for a particular period of the day. For example parking in residential areas may be vacant during office hours and thus can be utilized by others if required during this time. Initially different types of parking were used separately but over the time it was felt that sharing of parking could improve the performance of the guided management system. Moreover this sharing of parking spaces increases the available parking slots and thus results into better Parking Management System. Usage of communication technology is the backbone of the overall 
parking management as it provides all required information for parking suggestion.

The current paper is organized into various sections. Section I of the paper introduces the parking management system and discusses its basics. Architecture of the parking management system is elaborated in section II. Section III discusses the related work done in this area. This section also presents the usage of various techniques and their findings present in the literature. Section IV is dedicated to automated parking guidance system. This section further discusses the proposed modus operandi and discusses the various performance metrics. Simulation of the proposed work is presented in section $\mathrm{V}$ and it is observed that the proposed system outperforms existing approach.

\section{SySTEM ARChITECTURE OF GUIDED PARKING SySTEM}

The architecture of the proposed guided parking system consists of various components mainly sensors and communication devices. The status of each parking is monitored by a sensor device and communicated to the central server. This information is dynamic and is therefore monitored at regular intervals. All this information is available with central server, which is used for optimizing parking management. Whenever driver needs a parking, he initiates a request and specifies its destination. This request is sent to the server through a personal navigation device. Once the request is sent by the driver, it keeps on changing its position with respect to point of request. This change in position of the requesting vehicle is also forwarded to the central server. Additionally, the status of the parking is obtained through the sensor and transferred to the central server in real time. Based on the obtained information of the moving vehicle and parking facility, parking suggestion is made to the driver. The working of the automated parking management is illustrated in the following Fig.1. In the Fig.1 it is shown that the central server receives information from various parking facilities and stores this information in the server database. Central server also receives the request by a vehicle along with its position using GPS. Now as the requesting vehicle is moving continuously, the updated position of the vehicle is communicated to the central server at regular intervals.

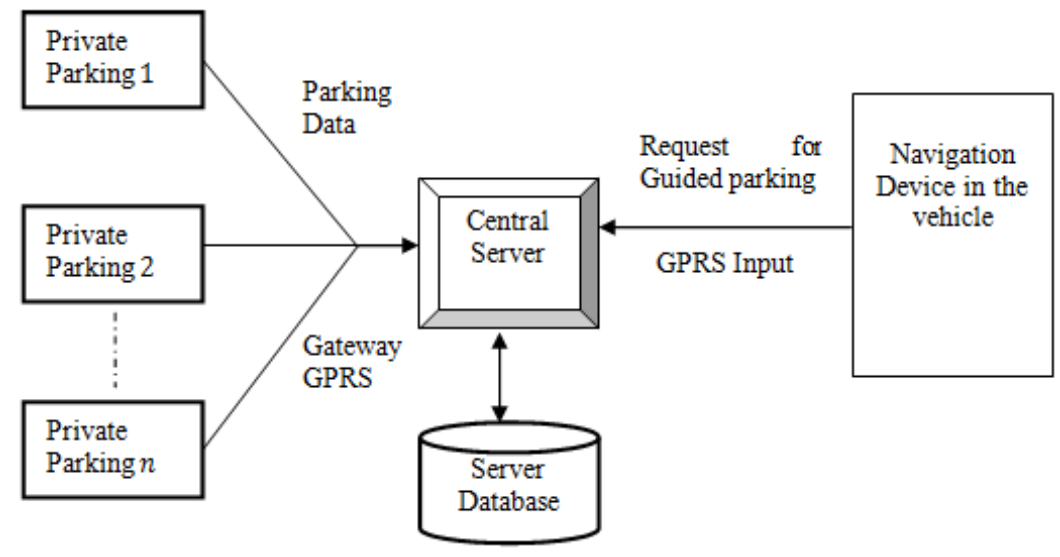

Fig.1. System architecture of Parking Guidance System

In the Fig. 1 it is shown that the central server receives information from various parking facilities and stores this information in the server database. Central server also receives the request by a vehicle along with its position using GPS. Now as the requesting vehicle is moving continuously, the updated position of the vehicle is communicated to the central server at regular intervals.

The automated parking guidance system considers various parking facilities in the city. Whenever the central server receives any request, the request is forwarded to the central server. Now central server, in association with the server database, suggests a parking to the requesting vehicle. The driver of the requesting vehicle in turn may reserve the parking or may even decline the suggestion. If driver declines the current suggestion, he may request again for which another best parking is suggested. The current position of the vehicle is obtained with the help of GPRS.

Many researchers have attempted the automated parking management using various approaches which have been discussed in the next section.

\section{RELATED WORK}

As discussed for the parking guidance system, dynamic information like number of vacant parking spots, location of the requesting car, parking fee is required. Recent advancement in ICT helps to obtain all required information for the guided parking system.

In earlier literature, parking information was obtained using image processing facility[1]. Over the time the method of obtaining parking information has been completely revolutionized. Various types of sensors are required to collect all required information and then communication networks helps to pass collected information to the central server [2][3]. Some authors have also suggested usage of combined sensor devices which are capable of sensing heterogeneous input signals [4][5] [6]. Thus there exists a wide range of sensors to 
choose from depending on the algorithm for a guided parking system.

Apart from sensor devices, major research focus is limited to the communication networks in the system. Various researchers have implemented different communication technologies. The authors in [7] have selected ZigBee protocol for its low installation cost. Authors in [8] implemented the wireless nodes. Authors in [9] and [10] implemented RF communication and IEEE 802.15.4 standards respectively. Vehicular ad hoc Network (VANET) is selected as an appropriate choice of communication in [11]. Authors in [12] have a vehicle to vehicle communication system where vehicles communicate with each other and exchange information, which can be used for various purpose like route determination. Thus there exists variety of communication technologies in the literature for the parking management system.

Despite the technical developments for data sensing and communication, its application to the parking management system is continuously evolving. Major research issue is the transfer of dynamic information to the driver and parking at regular intervals. Researchers are interested in providing better parking information to the drivers, so that it minimizes the total travelling distance. Some authors [13] [14] advocate communicating real time statistics so that drivers don't miss a parking spot while moving towards the parking spot suggested earlier. This change in the optimal parking spot results due to change in parameters over the time taken by driver to reach suggested parking. The authors in [15] emphasize on minimizing the parking fee. Authors in [11] have considered parking occupancy in future using the information collected from the VANETs for predicting the lot occupancy. Some researchers have focused on the behavioral aspect of the driver in the selection of parking [16][17]. Rodier and Shaheen [18] proposed E-parking which provides information to drivers during trip and allows them to reserve a parking spot. Authors in [19] suggest a parking to the driver based on the objective function. The driver may reserve the parking or may decline the suggested parking. In case of rejection by the driver, another parking is suggested to the driver. Hereafter, the subsequent subsection proposes an approach for automated parking guidance system.

\section{Automated PARKING GUIDANCE SYSTEM}

According to the related work in the domain, parking is suggested to the driver after evaluating various cost functions that represents the cost involved [20]. Similarly in the proposed methodology for Automated Parking Guidance System (APGS), authors propose two metric functions: availability metric and reservation metric. Function for suggestion metric evaluates the basis for suggesting a parking to the driver. Availability metric focuses on possibility of finding an empty parking spot when driver reaches to the parking. It is mainly evaluated based on various parameters including driving distance to the parking, walking distance of the parking to the destination, number of vacant slots etc. Furthermore reservation metric considers the cost of reserving a parking spot while driver reaches the suggested parking. It is therefore based on various parameters like parking cost, time taken by driver to reach suggested parking, condition of the path etc. In the proposed approach these metrics are evaluated for candidate parking and the one with lowest metric is selected as the best parking.

Availability metric is used for suggesting appropriate parking to the driver considering various input factors. It considers that the driver certainly gets available parking when he reaches the suggested parking. It is maintained by considering various concerned factors. Some of these factors are distance from parking to the destination, frequency of arrival and exit of vehicles from the parking, congestion along path to the suggested parking, parking cost etc.

The authors propose mathematical formulations for the availability metric. In order to present the mathematical formulations, few terms need to be defined as follows:

Average Time Between Arrival $(A T B A)$ represents the average frequency of arrival of vehicles in the parking. $A T B A$ helps in determining the number of cars that will be coming to the parking during the period while driver is driving towards the suggested parking. Similarly, we defined Average Time Between Exit $(A T B E)$ that represents how frequently vehicles exit from the parking. If $T_{d}$ represents the driving time to the suggested parking, then the number of cars coming to the parking $\left(n_{a}\right)$ and exiting from the parking $\left(n_{e}\right)$ during this period is calculated as:

$$
\begin{aligned}
& n_{a}=\frac{T_{d}}{A T B A} \\
& n_{e}=\frac{T_{d}}{A T B E}
\end{aligned}
$$

Thereafter, the degree of availability $\left(P_{a}\right)$ for the parking is defined as follows:

$$
P_{a}=\frac{n_{a}}{n+n_{e}} \quad \text { as }\left(\frac{\frac{T_{d}}{A T B A}}{n+\frac{T_{d}}{A T B E}}\right)
$$

where $n$ represents the number of vacant parking spots in the concerned parking.

Authors also take into consideration the situation when driver fails to get a vacant parking spot when he reaches the suggested parking $p_{s}$. In such situation, he requires driving to another parking which further increases the traffic and emissions. Therefore, it is taken into consideration that cost involving finding another parking remains minimal in case of failure. Therefore authors define an equation that minimizes the cost of finding next parking as follows:

$$
C=\sum \operatorname{dist}\left(p_{s}, p_{i}\right) * P_{i}, \text { for each } i \text { adj. to } s
$$

Where $\mathrm{p}_{\mathrm{i}}$ represents the parking adjacent to suggested 
parking $p_{s}$ and $P_{i}$ represents its degree of availability as defined above in Equation 3. The value of $\mathrm{C}$ represents the additional cost incurred if driver fails to get vacant slot at suggested parking. Therefore, parking with minimal $\mathrm{C}$ turns out to be a better option. Now after defining various parameters involved for finding suggestion metric $M_{s}$, we formulate the mathematical function defined as follows:

$$
M_{s}=\alpha_{1} T_{d}+\alpha_{2} W_{d}+\alpha_{3} P+\alpha_{4} P_{a}+\alpha_{5} C
$$

where

$\alpha s$ are the constant for each factor.

$T_{d}$ is travelling time taken from current location to parking.

$W_{d}$ is the walking distance from suggested parking to the destination.

$P$ is the parking cost.

$P_{a}$ is the probability of availability as given in Equation 3

$\mathrm{C}$ is the additional cost involved when it fails to get vacant slot at suggested parking.

Thereafter, as discussed above a driver may also reserve a parking spot in the suggested parking. Reservation of a parking spot guarantees availability of the parking spot after reaching the suggested parking although it incurs an additional cost. This additional cost is based on several parameters like travelling time during which the slot is kept reserved and the parking cost. The mathematical formulation for reservation metric $M_{r}$ is as follows:

$$
M_{r}=\beta_{1} T_{d}+\beta_{2} P+\beta_{3} W_{d}
$$

where

$\beta s$ are the constant for each factor.

$T_{d}$ is travelling time taken from current location of vehicle to destined parking.

$W_{d}$ is the walking distance from parking to the destination.

$P$ is the parking cost.

\section{A. Proposed Modus operandi}

This subsection discusses the modus operandi for parking management system after giving mathematical formulations for suggestion metric and reservation metric. Allocation of parking takes place in following steps:

Request for parking guidance: During this phase, the driver makes a request to the automated parking management system for parking guidance. The input for this phase is the destination which is specified by the driver. Sometimes the weight for each factor in metrics (suggestion and reservation) is also obtained from user based on his priorities. Additionally, the location of vehicle at request time is obtained through GPS and inputted to the system. All this input is sent to the central server which gives parking suggestion to the driver based on the information available with the server. Central server maintains the information about parking including parking charges, number of vacant spots etc., which is updated at regular intervals.

Determining best parking for reservation: using the input sent in the previous step and the suggestion metric as defined in Equation 5, the best candidate for reserving a parking spot is selected. Using the information received from the user and the information available with central server, appropriate parking is determined. The scrutinized parking is communicated to the requesting device. If the user wants to reserve the parking, he informs to reserve the parking spot until it reaches. The request for reservation is communicated to the central server and the concerned parking, which updates the status in response to this reservation. Thereafter, the user is guided regarding how to proceed towards the reserved parking.

Determining best parking for suggestion: if the user does not want to reserve the parking by the parking guidance system, he declines the reservation. In such case, the central server evaluates the suggestion metric $M_{S}$ as per Equation 5 for different parking options. The parking with lowest value of $M_{S}$ is suggested to the user. If the user decides to park in the suggested parking, user accepts the suggestion. Upon acceptance of suggestion, the status is updated and the user is guided with directions to reach the parking.

Updating parking spot status: After following the above steps, the guided car reaches the designated parking (reserved or not). As soon as the car reaches the parking, the parking spot status is updated and updated parameters are communicated to the central server. In case if driver fails to find the parking spot after reaching the parking, the driver may further request central server for parking guidance.

\section{B. Performance metrics for APGS}

The effectiveness of a parking guidance system may be evaluated with respect to various parameters. Some widely accepted parameters are as follows:

Average Driving Distance: This metric represents the averaged distance that a driver covers from its point the request to guided parking. The lower value of this metric is preferable for an effective parking as lower driving distance represents less emission of noise, smoke and other harmful effects.

Average Walking Distance: In addition to average walking distance, another most significant parameter is average walking distance. This represents the averaged distance that a user needs to walk to reach its destination.

Average level of congestion: The traffic congestion resulted by parking guidance is considered under this measure. It is measured by considering the number of cars heading to each parking. Lower value of this metric reflects that number of cars heading towards same parking is low, which is preferable as it ensures distributed parking.

Average occupancy rate of parking spots: Occupancy rate of parking spot is evaluated as ratio of occupied 
parking spot to the total number of spots. Thus this metric represents the utilization of parking resource. From its definition, it is evident that APGS should take efforts to maximize the average occupancy rate.

Average parking Failure rate: when a driver fails to find a vacant parking spot upon reaching the parking guided by APGS, his level of confidence, reliability and satisfaction in APGS decreases. Eventually drivers may not be interested to use APGS in future. Therefore it is important to minimize the average parking failure rate. It is calculated as the sum of all parking failures divided by the number of parking facilities.

Average requesting number: This metric represents the number of requests to APGS on average made by a driver until he succeeds in finding vacant parking slot. It is determined by finding ratio of all requests to the number of cars. An efficient APGS should aim to minimize the average requesting number.

\section{Simulation AND RESUlT}

Authors simulated the proposed methodology in MATLAB and the dataset for this implementation has been taken from the [20]. Authors have considered private, public and street parking in similar manner i.e. all different types of parking in [20] have been combined in one pool. In the simulation authors have taken 3 levels for the $\alpha s$ and $\beta s$ that represent the constant for function in suggestion metric and reservation metric as defined in Equation 5 and Equation 6 respectively. These 3 levels for constants of functions are low, medium and high which is represented by values 1,2 and 3 respectively. Authors assume that the parking request is generated as a uniform random distribution between 0 and 1 . To estimate distance, authors have considered rectilinear distance.

During the simulation, it remains a challenge to determine the values of constants to evaluate metric for suggestion and reservation as per Equation 5 and 6 respectively. Therefore, authors have considered various values (low, medium and high) for constants of the systems ( $\alpha s$ and $\beta s$ ) during different executions. In line to the simulation in [20], authors have also considered similar variations for constants known as base preference. The readers may refer to [20] to obtain the configuration of weight factors for constants in the suggestion metric and reservation metric. Authors have implemented the system for $N=500$. Following Table 1. Performance metrics for Guided parking ManagementTable 1 represents the values of different metrics which have been discussed earlier and are used to check effectiveness of a parking management system.

Table 1. Performance metrics for Guided parking Management

\begin{tabular}{cccccc}
\hline N & $\begin{array}{c}\text { Base } \\
\text { preference }\end{array}$ & $\begin{array}{c}\text { Avg. dis } \\
\text { (driving) } \\
\text { Km/car }\end{array}$ & $\begin{array}{c}\text { Avg. dis } \\
\text { (walking) } \\
\text { Km/driver }\end{array}$ & $\begin{array}{c}\text { Avg. occupancy } \\
\text { Per parking } \\
\text { (in \%age) }\end{array}$ & Avg. parking failure \\
\hline Case I-1 & I & 21.5605 & 0.9852 & 78.2173 & 0.01127 \\
$\begin{array}{c}\text { N= 500 } \\
\begin{array}{c}3803 \text { parking } \\
\text { Spots / }\end{array}\end{array}$ & II & 21.4662 & 0.9417 & 77.6274 & 0.01234 \\
61845 cars) & IV & 21.7290 & 0.9672 & $\mathbf{7 9 . 2 1 8 5}$ & 0.01092 \\
& V & 21.2202 & 0.9582 & 78.9123 & $\mathbf{0 . 0 1 0 6 3}$ \\
& VI & 21.2328 & 1.0012 & 78.5864 & 0.01147 \\
& VII & 21.1876 & $\mathbf{0 . 9 0 1 2}$ & 78.2143 & 0.01124 \\
& VIII & 21.4562 & 1.0028 & 78.1462 & 0.01232 \\
\hline \hline $\begin{array}{c}\text { Optimized value } \\
\text { Of the metric }\end{array}$ & & $\mathbf{2 1 . 1 1 8 5}$ & $\mathbf{0 . 9 0 1 2}$ & $\mathbf{0 . 9 0 1 2}$ & 0.01108 \\
\hline
\end{tabular}

In Table $1, N$ represents the number of parking requests generated during the period of simulation. Here it is observed that the optimized value of performance metrics in the proposed approach outperforms the existing approach [20]. These optimized values of performance metric are better than the best obtained values. The major contribution of the proposed approach which helps in outperforming the existing approach is the inclusion of average time between exits of vehicles from parking. This parameter ATBE helps in forecasting the number of vacant parking spots in future for a parking. Thus by estimating the number of empty parking spots, central server in the parking management system can efficiently suggest a parking to the requester. The comparison of proposed approach with existing solution has been represented in following graphs.

In the existing approach, public and private parking have been considered separately. It is observed that although the occupancy rate of public parking is pretty good while for private parking, it needs to be strongly worked upon. In the proposed approach, authors have considered both parking (public and private) uniformly that resulted in the significant improvement in the occupancy rate of the parking. During simulation, it is 
noticed that the average occupancy rate of proposed approach is $78.49 \%$ while for existing approach, average occupancy rate is merely $57.33 \%$. Thus the proposed approach improves the occupancy rate of parking by remarkable margin. This remarkable improvement in the average occupancy rate of the parking is obtained by considering the ATBE during suggesting a parking to the requesting vehicle.

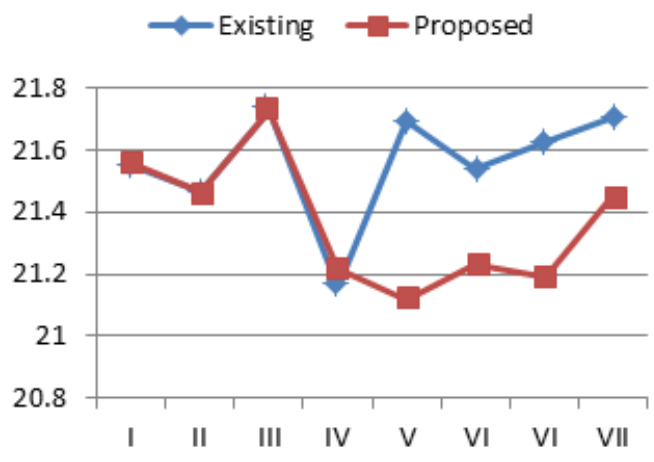

Fig.2. Comparison of Average Driving distance
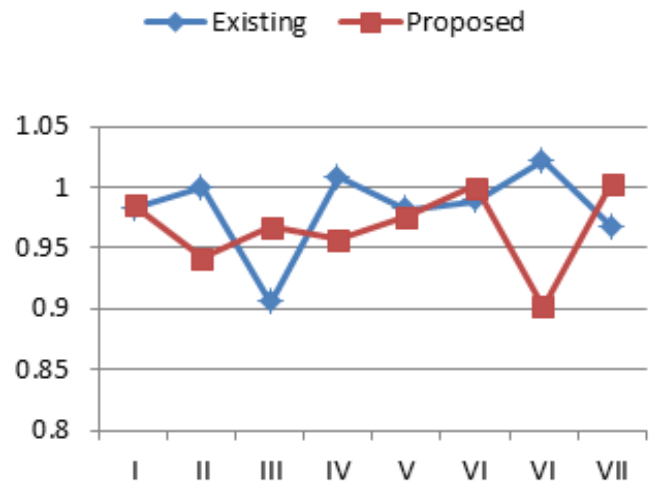

Fig.3. Comparison of Average Walking distance

The comparison of average driving and walking distance is shown in Fig.2 and Fig.3 respectively. From the graphical representation, it is evident that the proposed approach outperforms the existing approach.

\section{CONCLUSION}

In this paper, authors have proposed a guided parking management system for megacities. In the proposed approach, two metrics have been suggested and formulated. The first metric for suggestion is used to suggest a parking to the requesting vehicle. This suggestion is determined based on destination, current position of the vehicle, parking charges etc. Another metric for reservation aims to provide a parking which will be reserved until car reaches the parking. This ensures the availability of vacant spot when car reaches the suggested parking. Thus it further reduces the overall traffic/ congestion on the roads which is caused due to unavailability of vacant parking spot upon reaching suggested parking. Average time between exits ( $A T B E$ ) and Average time between arrivals $(A T B A)$ have been considered in formulating these metrics in addition to several other factors

The proposed methodology has been implemented in MATLAB. It is already mentioned that in the current implementation, authors have considered all parking facilities in similar manner. The various metrics to evaluate effectiveness of parking management system have already been discussed above. Authors have evaluated the effectiveness of the proposed approach in terms of mentioned metrics i.e. average driving distance, average walking distance, occupancy of parking etc. During implementation it is proved that the proposed implementation outperforms the existing results in terms of the optimized solutions. The proposed methodology generates better optimized solutions than the existing results. We have Hence the proposed methodology can be accepted as an efficient and effective approach for automated parking management systems.

\section{REFERENCES}

[1] M. O. Reza, M. F. Ismail, A. A. Rokoni, and M. A. R. Sarkar, "Smart Parking System with Image Processing Facility," no. April, pp. 41-47, 2012.

[2] S. Yiu Cheung, S. Coleri Ergen, and P. Varaiya, "Traffic Surveillance with Wireless Magnetic Sensors."

[3] M. Y. I. Idris, E. M. Tamil, N. M. Noor, Z. Razak, and K. W. Fong, "Parking guidance system utilizing wireless sensor network and ultrasonic sensor," Information Technology Journal, vol. 8, no. 2. pp. 138-146, 2009.

[4] J. Chinrungrueng, U. Sunantachaikul, and S. Triamlumlerd, "Smart parking: An application of optical wireless sensor network," in Applications and the Internet Workshops, 2007. SAINT Workshops 2007. International Symposium on, 2007, p. 66.

[5] V. W. S. Tang, Y. Zheng, and J. Cao, "An intelligent car park management system based on wireless sensor networks," in Pervasive Computing and Applications, 2006 1st International Symposium on, 2006, pp. 65-70.

[6] S. Lee, D. Yoon, and A. Ghosh, "Intelligent parking lot application using wireless sensor networks," in Collaborative Technologies and Systems, 2008. CTS 2008. International Symposium on, 2008, pp. 48-57.

[7] S. Shim, S. Park, and S. Hong, "Parking management system using zigbee," Int. J. Comput. Sci. Netw. Secur., vol. 6, pp. 131-137, 2006.

[8] V. K. Boda, A. Nasipuri, and I. Howitt, "Design considerations for a wireless sensor network for locating parking spaces," in SoutheastCon, 2007. Proceedings. IEEE, 2007, pp. 698-703.

[9] S. V Srikanth, P. J. Pramod, K. P. Dileep, S. Tapas, M. U. Patil, and others, "Design and implementation of a prototype smart PARKing (SPARK) system using wireless sensor networks," in Advanced Information Networking and Applications Workshops, 2009. WAINA'09. International Conference on, 2009, pp. 401406.

[10] D. Silva, P. Bartolomeu, and J. Fonseca, "Wireless parking lot monitoring and guidance," in Emerging Technologies \& Factory Automation (ETFA), 2011 IEEE 16th Conference on, 2011, pp. 1-4.

[11] M. Caliskan, A. Barthels, B. Scheuermann, and M. Mauve, "Predicting parking lot occupancy in vehicular ad hoc networks," in Vehicular Technology Conference, 2007. VTC2007-Spring. IEEE 65th, 2007, pp. 277-281.

[12] R. N. Minihi and H. M. Alsabbagh, "Analytical Model for 
Wireless Channel of Vehicle-to-Vehicle Communications," no. July, pp. 48-59, 2017.

[13] D. Teodorović and P. Lučić, "Intelligent parking systems," Eur. J. Oper. Res., vol. 175, no. 3, pp. 1666-1681, 2006.

[14] B. J. Waterson, N. B. Hounsell, K. Chatterjee, and others, "Quantifying the potential savings in travel time resulting from parking guidance systems-a simulation case study," J. Oper. Res. Soc., vol. 52, no. 10, pp. 1067-1077, 2001.

[15] S.-Y. Chou, S.-W. Lin, and C.-C. Li, "Dynamic parking negotiation and guidance using an agent-based platform," Expert Syst. Appl., vol. 35, no. 3, pp. 805-817, 2008.

[16] P. Bonsall and I. Palmer, "Modelling drivers' car parking behaviour using data from a travel choice simulator," Transp. Res. Part C Emerg. Technol., vol. 12, no. 5, pp. 321-347, 2004.

[17] M. E. I. Zhenyu, Y. XIANG, C. Jun, and W. Wei, "Optimizing model of curb parking pricing based on parking choice behavior," J. Transp. Syst. Eng. Inf. Technol., vol. 10, no. 1, pp. 99-104, 2010.

[18] C. J. Rodier and S. A. Shaheen, "Transit-based smart parking: An evaluation of the San Francisco bay area field test," Transp. Res. Part C Emerg. Technol., vol. 18, no. 2, pp. 225-233, 2010.

[19] Y. Geng and C. G. Cassandras, "A new 'smart parking' system based on optimal resource allocation and reservations," in Intelligent Transportation Systems (ITSC), 2011 14th International IEEE Conference on, 2011, pp. 979-984.

[20] J.-H. Shin, N. Kim, H.-B. Jun, and D. Y. Kim, “A Dynamic Information-Based Parking Guidance for Megacities considering Both Public and Private Parking," 2017.

\section{Authors' Profiles}

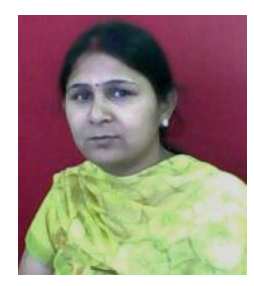

Monika Mangla has more than 15 years of teaching experience. Currently she is pursuing research at Thapar University and working as Assistant Professor in the Department of Computer Engineering, Mumbai University. She has many research papers published in reputed conferences and journal. Her area of specialization is algorithms, computational geometry, optimization etc.

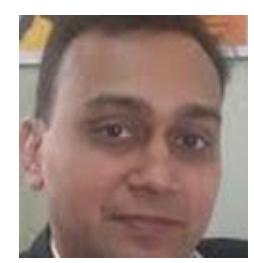

Deepak Garg has done his Ph.D. in efficient algorithm design from Thapar University. He has 20 years of rich experience in engineering education. He is Senior Member of IEEE (Institute of Electrical and Electronics Engineers), USA, Executive Member of IEEE Delhi Section and secretary of IEEE Computer Society, Delhi Section. He is Life Member of ISTE, CSI, IETE (Institute of Electronics and telecommunication Engineers), ISC (Indian Science Congress), British Computer Society and ACM, UK. He is currently working as Professor and Head, Bennett University. He has several research papers in reputed journals and conferences to this credit. He is on the Editorial Board of many International Journals. His active research area is Algorithms and Data Mining, deep learning and machine learning.
How to cite this paper: Monika Mangla, Deepak Garg, "An Automated Parking Guidance System for Megacities", International Journal of Information Technology and Computer Science(IJITCS), Vol.10, No.1, pp.52-58, 2018. DOI: 10.5815/ijitcs.2018.01.06 